Article

\title{
Homomorphic Image and Inverse Image of Weak Closure Operations on Ideals of BCK-Algebras
}

\author{
Hashem Bordbar ${ }^{1}$, Young Bae Jun ${ }^{2}$ and Seok-Zun Song ${ }^{3, *}$ \\ 1 Center for Information Technologies and Applied Mathematics, University of Nova Gorica, \\ 5000 Nova Gorica, Vipavska 13, Slovenia; Hashem.bordbar@ung.si \\ 2 Department of Mathematics Education, Gyeongsang National University, Jinju 52828, Korea; \\ skywine@gmail.com \\ 3 Department of Mathematics, Jeju National University, Jeju 690-756, Korea \\ * Correspondence: szsong@jejunu.ac.kr
}

Received: 25 February 2020; Accepted: 2 April 2020; Published: 11 April 2020

check for updates

\begin{abstract}
We introduce the notions of meet, semi-prime, and prime weak closure operations. Using homomorphism of BCK-algebras $\varphi: X \rightarrow Y$, we show that every epimorphic image of a non-zeromeet

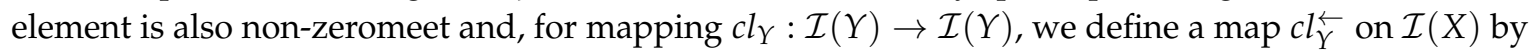
$A \mapsto \varphi^{-1}\left(\varphi(A)^{c l_{Y}}\right)$. We prove that, if " $c l_{Y}$ " is a weak closure operation (respectively, semi-prime and meet) on $\mathcal{I}(Y)$, then so is " $c l_{Y}^{\leftarrow}$ " on $\mathcal{I}(X)$. In addition, for mapping $c l_{X}: \mathcal{I}(X) \rightarrow \mathcal{I}(X)$, we define a map $c l_{X}$ on $\mathcal{I}(Y)$ as follows: $B \mapsto \varphi\left(\varphi^{-1}(B)^{c l_{X}}\right)$. We show that, if " $c l_{X}$ " is a weak closure operation (respectively, semi-prime and meet) on $\mathcal{I}(X)$, then so is " $c l_{X}{ }^{\prime}$ " on $\mathcal{I}(Y)$.
\end{abstract}

Keywords: zeromeet element; meet ideal; relative annihilator; semi-prime map; meet map; (semi-prime, meet) weak closure operation

\section{Introduction}

Imai and Iseki introduced the concept of BCK-algebra in 1966. BCK-algebra is a generalization of set-theoretic difference. In addition, in [1], Bordbar et al. introduced the concept of weak closure operation as a generalization of closure operation on ideals of a BCK-algebras. They investigated some properties of weak closure operations and provided examples. They defined many different types of weak closure operations on ideals of an arbitrary $B C K$-algebra such that finite type, (strong) semi-primeness, tender, and naive weak closure operations and investigated their related properties [2-5]. Besides, the notions of commutative, implicative, and positive implicative weak closure operations were investigated by [1]. Moreover, using the notation of weak closure operation, some algebraic structures have been defined and related properties investigated [6-8].

In this article, the notions of semi-prime, meet, and prime weak closure operation are introduced, and their relations and properties investigated. Using an epimorphism $\varphi: X \rightarrow Y$ of lower BCK-semilattices $X$ and $Y$, mapping $c l_{Y}: \mathcal{I}(Y) \rightarrow \mathcal{I}(Y)$, and any ideal $A$ of $X$ with $\operatorname{ker}(\varphi) \subseteq A$, we define a new map $c l_{Y}^{\leftarrow}$ on $\mathcal{I}(X)$ by

$$
A \mapsto \varphi^{-1}\left(\varphi(A)^{c l_{Y}}\right)
$$

and investigate related properties of this map. We show that, if " $c l_{Y}$ " is a weak closure operation (respectively, semi-prime, meet, and prime) on $\mathcal{I}(Y)$, then so is " $c l_{Y}^{\leftarrow}$ " on $\mathcal{I}(X)$.

Similarly, using a map $c l_{X}: \mathcal{I}(X) \rightarrow \mathcal{I}(X)$ and any ideal $B$ of $Y$, we define a new map $c l_{X}$ on $\mathcal{I}(Y)$ as follows:

$$
B \mapsto \varphi\left(\varphi^{-1}(B)^{c l_{X}}\right)
$$


We prove that, if " $c l_{X}$ " is a weak closure operation (respectively, semi-prime, meet, and prime) on $\mathcal{I}(X)$, then so is " $c l \vec{X}$ " on $\mathcal{I}(Y)$.

\section{Preliminaries}

$B C I / B C K$-algebra, which was introduced by K. Iséki, is a class of logical algebras. In what follows, we gather some necessary definitions and theorems which we need for further results.

An algebra $(X ; *, 0)$ of type $(2,0)$ is called a BCI-algebra if it satisfies the following conditions:

(I) $(\forall x \in X)(x * x=0)$;

(II) $\quad(\forall x, y \in X)((x *(x * y)) * y=0)$;

(III) $\quad(\forall x, y, z \in X)(((x * y) *(x * z)) *(z * y)=0)$; and

(IV) $(\forall x, y \in X)(x * y=0, y * x=0 \Rightarrow x=y)$.

Moreover, if an arbitrary $B C I$-algebra $X$ satisfies the following condition, then $X$ is called a $B C K$-algebra:

(V) $\quad(\forall x \in X)(0 * x=0)$.

Lemma 1. Suppose that $X$ is a BCI/BCK-algebra. Then, the following axioms are satisfied:

(a1) $(\forall x \in X)(x * 0=x)$;

(a2) $(\forall x, y, z \in X)(x \leq y \Rightarrow x * z \leq y * z, z * y \leq z * x)$;

(a3) $\quad(\forall x, y, z \in X)((x * y) * z=(x * z) * y$; and

(a4) $\quad(\forall x, y, z \in X) .((x * z) *(y * z) \leq x * y)$

We define $\leq$ such that $x * y=0$. if and only if $x \leq y$, and call it BCK-order.

For a $B C K$-algebra $X$, if $X$ concerning the $B C K$-order is a lower semilattice, then we called it the lower BCK-semilattice.

Definition 1. Suppose that $X$ is a BCK-algebra and $A$ is a subset of $X . A$ is called an ideal of $X$ when the following assertions hold:

$$
\begin{aligned}
& 0 \in A, \\
& (\forall x \in X)(\forall y \in A)(x * y \in A \Rightarrow x \in A) .
\end{aligned}
$$

The following condition is satisfied for any ideal A of a BCK-algebra X

$$
(\forall x, y \in X)(x \leq y, y \in A \Rightarrow x \in A) .
$$

Remark 1. Let $A$ be a subset of $X$. The intersection of all ideals of $X$ containing $A$ is an ideal of $X$ and it is denoted by $\langle A\rangle$. Moreover, If $A$ is a finite set, then we say that $\langle A\rangle$ is finitely generated ideal of $X$.

From now on, $X$ and $\mathcal{I}(X)$ are the lower $B C K$-semilattice and the set of all ideals of the $X$, respectively, unless otherwise specified. In addition, for more information regarding the ordered algebra and $B C I / B C K$-algebras, please refer to the books $[9,10]$.

Definition 2 ([1]). Suppose that $X$ is a lower BCK-semilattice and $x \in X$. Then, the element $x$ is called a zeromeet element of $X$ if the following condition holds.

$$
(\exists y \in X \backslash\{0\})(x \wedge y=0) .
$$

Otherwise, $x$ is called a non-zeromeet element of $X$.

The set of all zeromeet elements of $X$ is denoted by $Z(X)$ and defined as follow: 


$$
Z(X)=\{x \in X \mid x \wedge y=0 \text { for some nonzero element } y \in X\} .
$$

It is clear that $0 \in Z(X)$ and also, for the greatest element 1 , we have $1 \in X \backslash Z(X)$.

Lemma 2 ([1]). The set $X \backslash Z(X)$ is closed under the $\wedge$ operation.

Definition 3 ([6]). Let $A$ and $B$ be nonempty subsets of $X$; define $A \wedge B$ as follows:

$$
A \wedge B:=\langle\{a \wedge b \mid a \in A, b \in B\}\rangle ;
$$

and call it the meet ideal of $X$ generated by $A$ and $B$. Furthermore, $\{a\} \wedge B$ is denoted by $a \wedge B$ and, similarly, $A \wedge\{b\}$ is denoted by $A \wedge b$.

Theorem 1 ([6]). If $A$ and $B$ are ideals of $X$, then the set

$$
A \wedge B=\{a \wedge b \mid a \in A, b \in B\}
$$

is an ideal of $X$ based on $A$ and $B$.

Definition 4 ([8]). Define a set $(A: \wedge B)$ as follow for nonempty subsets $A$ and $B$ of $X$ and call it the relative annihilator of $B$ with respect to $A$.

$$
(A: \wedge B):=\{x \in X \mid x \wedge B \subseteq A\}
$$

Lemma 3 ([8]). The relative annihilator $(A: \wedge B)$ of $B$ with respect to $A$ is an ideal of $X$, where $A$ and $B$ are ideals of $X$.

Lemma 4 ([8]). For ideal $A$ of $X$, it is clear that $\left(A:_{\wedge} X\right)=A$ and $(A: \wedge A)=X$.

Now, we define a weak closure operation.

Definition 5 ([1]). A weak closure operation on set of ideals of a BCK-algebra is defined as a mapping $c l: \mathcal{I}(X) \rightarrow \mathcal{I}(X)$, if the following conditions hold.

$$
\begin{aligned}
& (\forall A \in \mathcal{I}(X))(A \subseteq \operatorname{cl}(A)), \\
& (\forall A, B \in \mathcal{I}(X))(A \subseteq B \Rightarrow \operatorname{cl}(A) \subseteq \operatorname{cl}(B)) .
\end{aligned}
$$

For a weak closure operation $c l$, ff the condition

$$
(\forall A \in \mathcal{I}(X))(\operatorname{cl}(\operatorname{cl}(A))=\operatorname{cl}(A)),
$$

holds, then we call it a closure operation on $\mathcal{I}(X)$ (see [3]).

For simplicity, we write $A^{c l}$ instead of $c l(A)$.

Definition 6 ([5]). A semi-prime weak closure operation " $c l$ " on $\mathcal{I}(X)$ is defined as follows:

$$
(\forall A, B \in \mathcal{I}(X))\left(A \wedge B^{c l} \subseteq(A \wedge B)^{c l}\right) .
$$

Definition 7 ([5]). A meet weak closure operation " $c l$ " on $\mathcal{I}(X)$ is defined as follows:

$$
(\forall A \in \mathcal{I}(X))(\forall a \in X \backslash Z(X))\left((a \wedge A)^{c l}=a \wedge A^{c l}\right) .
$$


Definition 8. A prime weak closure operation "cl" on $\mathcal{I}(X)$ is a semi-prime and meet weak closure operation on $\mathcal{I}(X)$.

Lemma 5 ([9]). If $\varphi: X \rightarrow Y$ is an epimorphism of lower BCK-semilattices, then

$$
(\forall x, y \in X)\left(\varphi\left(x \wedge_{X} y\right)=\varphi(x) \wedge_{Y} \varphi(y)\right)
$$

\section{Homomorphic (Inverse) Images of (Meet, Semi-Prime, and Prime) Weak Closure Operations}

Definition 9. Let $c l: \mathcal{I}(X) \rightarrow \mathcal{I}(X)$ be a map on set of ideals of a BCK-algebra X. This map is said to be meet when the following condition holds:

$$
(\forall A \in \mathcal{I}(X))(\forall a \in X \backslash Z(X))\left((a \wedge A)^{c l}=a \wedge A^{c l}\right)
$$

Example 1. Suppose that $X=\{0,1,2,3,4\}$ with the following Cayley table:

\begin{tabular}{l|lllll}
$*$ & 0 & 1 & 2 & 3 & 4 \\
\hline 0 & 0 & 0 & 0 & 0 & 0 \\
1 & 1 & 0 & 1 & 0 & 0 \\
2 & 2 & 2 & 0 & 0 & 0 \\
3 & 3 & 3 & 3 & 0 & 0 \\
4 & 4 & 4 & 4 & 3 & 0
\end{tabular}

Then, $X$ is a lower $B C K$-semilattice with five following ideals: $A_{0}=\{0\}, A_{1}=\{0,1\}, A_{2}=\{0,2\}$, $A_{3}=\{0,1,2\}$ and $A_{4}=X$ and also $Z(X)=\{0,1,2\}$. Now, define a map "cl" on $\mathcal{I}(X)$ such that $A_{0}^{c l}=A_{0}$, $A_{1}^{c l}=A_{2}, A_{2}^{c l}=A_{1}, A_{3}^{c l}=A_{4}$ and $A_{4}^{c l}=A_{3}$. It is routine to verify that "cl" is a meet map on $\mathcal{I}(X)$. However, "cl" is not a weak closure operation since $A_{1} \nsubseteq A_{2}=A_{1}^{c l}$.

By a meet weak closure operation on $\mathcal{I}(X)$ (see [5]), we mean a weak closure operation $c l$ on $\mathcal{I}(X)$ which satisfies the condition in Equation (10).

Definition 10. A map $c l: \mathcal{I}(X) \rightarrow \mathcal{I}(X)$ is called semi-prime if the following condition holds:

$$
(\forall A, B \in \mathcal{I}(X))\left(A \wedge B^{c l} \subseteq(A \wedge B)^{c l}\right)
$$

Example 2. Suppose that $X=\{0,1,2,3,4\}$ with the following Cayley table:

\begin{tabular}{l|lllll}
$*$ & 0 & 1 & 2 & 3 & 4 \\
\hline 0 & 0 & 0 & 0 & 0 & 0 \\
1 & 1 & 0 & 0 & 0 & 1 \\
2 & 2 & 1 & 0 & 0 & 2 \\
3 & 3 & 1 & 1 & 0 & 3 \\
4 & 4 & 4 & 4 & 4 & 0
\end{tabular}

Then, $X$ is a lower $B C K$-semilattice with four ideals: $A_{0}=\{0\}, A_{1}=\{0,1,2,3\}, A_{2}=\{0,4\}$ and $A_{3}=X$. Now, define a map " $c l$ " on $\mathcal{I}(X)$ such that $A_{0}^{c l}=A_{3}, A_{1}^{c l}=A_{2}, A_{2}^{c l}=A_{1}$, and $A_{3}^{c l}=A_{0}$. Then, "cl" is a semi-prime map on set of ideals of $X$. However, it is not a weak closure operation because $A_{1} \nsubseteq A_{2}=A_{1}^{c l}$.

By a semi-prime weak closure operation on $\mathcal{I}(X)$ (see [5]), we mean a weak closure operation $\mathrm{cl}$ on $\mathcal{I}(X)$, which satisfies the condition in Equation (11).

The notions of semi-prime map and meet map are independent. The following examples show it. 
Example 3. Let $X$ be a set of $\{0,1,2,3,4\}$ with the following Cayley table:

\begin{tabular}{l|lllll}
$*$ & 0 & 1 & 2 & 3 & 4 \\
\hline 0 & 0 & 0 & 0 & 0 & 0 \\
1 & 1 & 0 & 1 & 0 & 0 \\
2 & 2 & 2 & 0 & 0 & 0 \\
3 & 3 & 3 & 3 & 0 & 0 \\
4 & 4 & 3 & 4 & 1 & 0
\end{tabular}

Then, by routine verification, $X$ is a $B C K$-semilattice and $Z(X)=\{0,1,2\}$. In addition, $X$ has five ideals: $A_{0}=\{0\}, A_{1}=\{0,1\}, A_{2}=\{0,2\}, A_{3}=\{0,1,2\}$ and $A_{4}=X$. Now, define a map "cl" on $\mathcal{I}(X)$ by $A_{0}^{c l}=A_{0}, A_{1}^{c l}=A_{2}, A_{2}^{c l}=A_{1}, A_{3}^{c l}=A_{4}$ and $A_{4}^{c l}=A_{4}$. Then, "cl" is a meet map on $\mathcal{I}(X)$. However, "cl" is not a semi-prime map on $\mathcal{I}(X)$ since $A_{1} \wedge A_{2}^{c l}=A_{1} \wedge A_{1}=A_{1} \nsubseteq A_{0}=A_{0}^{c l}=\left(A_{1} \wedge A_{2}\right)^{c l}$.

Example 4. Let $X$ be a set of $\{0,1,2,3,4\}$ with the following Cayley table:

\begin{tabular}{l|lllll}
$*$ & 0 & 1 & 2 & 3 & 4 \\
\hline 0 & 0 & 0 & 0 & 0 & 0 \\
1 & 1 & 0 & 0 & 0 & 0 \\
2 & 2 & 2 & 0 & 0 & 2 \\
3 & 3 & 3 & 2 & 0 & 3 \\
4 & 4 & 4 & 4 & 4 & 0
\end{tabular}

Then, by routine verification, $X$ is a $B C K$-semilattice and $Z(X)=\{0\}$. In addition, $X$ has five ideals: $A_{0}=\{0\}, A_{1}=\{0,1\}, A_{2}=\{0,1,2,3\}, A_{3}=\{0,1,4\}$ and $A_{4}=X$. Now, define "cl" on $\mathcal{I}(X)$ by $A_{0}^{c l}=A_{1}, A_{1}^{c l}=A_{4}, A_{2}^{c l}=A_{4}, A_{3}^{c l}=A_{3}$, and $A_{4}^{c l}=A_{4}$. Then, "cl" is a semi-prime map on $\mathcal{I}(X)$ which is not meet since

$$
3 \wedge A_{2}^{c l}=3 \wedge A_{4}=A_{2} \neq A_{4}=A_{2}^{c l}=\left(3 \wedge A_{2}\right)^{c l}
$$

In the following examples, we see that a meet weak closure operation is independent of a semi-prime weak closure operation.

Example 5. Suppose that $X=\{0,1,2,3,4\}$. Then, $X$ is a lower BCK-semilattice with the following Cayley table:

\begin{tabular}{l|lllll}
$*$ & 0 & 1 & 2 & 3 & 4 \\
\hline 0 & 0 & 0 & 0 & 0 & 0 \\
1 & 1 & 0 & 0 & 1 & 0 \\
2 & 2 & 1 & 0 & 2 & 0 \\
3 & 3 & 3 & 3 & 0 & 0 \\
4 & 4 & 4 & 4 & 4 & 0
\end{tabular}

We also have $Z(X)=\{0,1,2,3\}$ and $X$ has five ideals: $A_{0}=\{0\}, A_{1}=\{0,1,2\}, A_{2}=\{0,3\}, A_{3}=$ $\{0,1,2,3\}$, and $A_{4}=X$. Now, define "cl" on $\mathcal{I}(X)$ by $A_{0}^{c l}=A_{1}, A_{1}^{c l}=A_{3}, A_{2}^{c l}=A_{3}, A_{3}^{c l}=A_{3}$ and $A_{4}^{c l}=A_{4}$. Then, "cl" is a meet weak closure operation on $\mathcal{I}(X)$ which is not a semi-prime map since

$$
A_{1}^{c l} \wedge A_{2}=A_{3} \wedge A_{2}=A_{2} \nsubseteq A_{1}=A_{0}^{c l}=\left(A_{1} \wedge A_{2}\right)^{c l} .
$$

Example 6. Consider $X=\{0,1,2,3,4\}$ the same as given in Example 4. If we define "cl" on $\mathcal{I}(X)$ by $A_{0}^{c l}=A_{1}, A_{1}^{c l}=A_{4}, A_{2}^{c l}=A_{4}, A_{3}^{c l}=A_{4}$ and $A_{4}^{c l}=A_{4}$, then "cl" is a weak closure operation which is semi-prime but not meet since

$$
4 \wedge A_{2}^{c l}=4 \wedge A_{4}=A_{3} \neq A_{4}=\left(4 \wedge A_{2}\right)^{c l} .
$$

Definition 11. A prime map $c l: \mathcal{I}(X) \rightarrow \mathcal{I}(X)$ is a map which is both meet and semi-prime. 
Example 7. Let $X=\{0,1,2,3\}$ and define the following Cayley table:

\begin{tabular}{l|llll}
$*$ & 0 & 1 & 2 & 3 \\
\hline 0 & 0 & 0 & 0 & 0 \\
1 & 1 & 0 & 1 & 0 \\
2 & 2 & 2 & 0 & 0 \\
3 & 3 & 3 & 3 & 0
\end{tabular}

Then, $X$ is a lower $B C K$-semilattice and, clearly, $Z(X)=\{0,1,2\}$. In addition, $X$ has five ideals: $A_{0}=\{0\}$, $A_{1}=\{0,1\}, A_{2}=\{0,2\}, A_{3}=\{0,1,2\}$, and $A_{4}=X$. Now, define a map "cl" on $\mathcal{I}(X)$ by $A_{0}^{c l}=A_{4}$, $A_{1}^{c l}=A_{3}, A_{2}^{c l}=A_{3}, A_{3}^{c l}=A_{3}$ and $A_{4}^{c l}=A_{4}$. Then, "cl" is a prime map on $\mathcal{I}(X)$.

Remember that, by a prime weak closure operation, we mean a weak closure operation $c l$ on set of ideals, which is both meet and semi-prime.

Example 8. Let $X=\{0,1,2,3,4\}$ with the conditions given in Example 5. Then, $X$ has five ideals: $A_{0}=\{0\}$, $A_{1}=\{0,1,2\}, A_{2}=\{0,3\}, A_{3}=\{0,1,2,3\}$, and $A_{4}=X$. In addition, $Z(X)=\{0,1,2,3\}$. If we define "cl" on $\mathcal{I}(X)$ by $A_{0}^{c l}=A_{1}, A_{1}^{c l}=A_{1}, A_{2}^{c l}=A_{3}, A_{3}^{c l}=A_{4}$, and $A_{4}^{c l}=A_{4}$, then " $c l$ " is a prime weak closure operation on $\mathcal{I}(X)$.

Lemma 6 ([9]). If $\varphi: X \rightarrow Y$ is an epimorphism of lower BCK-semilattices, then

$$
(\forall x, y \in X)\left(\varphi\left(x \wedge_{X} y\right)=\varphi(x) \wedge_{Y} \varphi(y)\right)
$$

Theorem 2. Every isomorphic image of a non-zeromeet element is also non-zeromeet.

Proof. Let $\varphi: X \rightarrow Y$ be an isomorphism of lower $B C K$-semilattices. Suppose that $z$ is a non-zeromeet element of $X$. Let $y \in Y$ be such that $y \wedge_{Y} \varphi(z)=0$. Then, $y=\varphi(x)$ for some $x \in X$, and thus

$$
0=y \wedge_{Y} \varphi(z)=\varphi(x) \wedge_{Y} \varphi(z)=\varphi\left(x \wedge_{X} z\right)
$$

by using Lemma 6. Hence, $x \wedge_{X} z \in \operatorname{ker}(\varphi)=\{0\}$, and thus $x \wedge_{X} z=0$. Since $z$ is a non-zeromeet element of $X$, it follows that $x=0$ and thus $y=\varphi(x)=\varphi(0)=0$. Therefore, $\varphi(z)$ is a non-zeromeet element of $Y$.

Lemma 7 ([9]). $\quad$ 1. Let $\varphi: X \rightarrow Y$ be an epimorphism of $B C K$-algebras. If $A$ is an ideal of $X$, then $\varphi(A)$ is an ideal of $Y$.

2. Let $\varphi: X \rightarrow Y$ be a homomorphism of BCK-algebras. If $B$ is an ideal of $Y$, then $\varphi^{-1}(B)$ is an ideal of $X$.

Lemma 8 ([9]). Let $\varphi: X \rightarrow Y$ be a homomorphism of BCK-algebras $X$ and $Y$ and let $A$ be an ideal of $X$ such that $\operatorname{Ker}(\varphi) \subseteq A$. Then, $\varphi^{-1}\left(A^{\prime}\right)=A$ where $A^{\prime}=\varphi(A)$.

Lemma 9. If $\varphi: X \rightarrow Y$ is a homomorphism of lower BCK-semilattices, then

$$
\varphi^{-1}(G) \wedge_{X} \varphi^{-1}\left(H^{c l_{Y}}\right) \subseteq \varphi^{-1}\left(G \wedge_{Y} H^{c l_{Y}}\right)
$$

for all ideals $G$ and $H$ of $Y$. Moreover, if $\varphi$ is an isomorphism, then

$$
\varphi^{-1}\left(G \wedge_{Y} H^{c l_{Y}}\right)=\varphi^{-1}(G) \wedge_{X} \varphi^{-1}\left(H^{c l_{Y}}\right)
$$

for all ideals $G$ and $H$ of $Y$. 
Proof. Let $G$ and $H$ be ideals of $Y$. Assume that $\varphi: X \rightarrow Y$ is a homomorphism of lower $B C K$-semilattices. If $z \in \varphi^{-1}(G) \wedge_{X} \varphi^{-1}\left(H^{c l_{Y}}\right)$, then there exist $x \in \varphi^{-1}(G)$ and $y \in \varphi^{-1}\left(H^{c l_{Y}}\right)$ such that $z=x \wedge_{X} y$. Hence,

$$
\varphi(z)=\varphi\left(x \wedge_{X} y\right)=\varphi(x) \wedge_{Y} \varphi(y) \in G \wedge_{Y} H^{c l_{Y}} .
$$

Therefore, $z \in \varphi^{-1}\left(G \wedge_{Y} H^{c^{c l}}\right)$.

Now, suppose that $\varphi: X \rightarrow Y$ is an isomorphism of lower $B C K$-semilattices and let $z \in \varphi^{-1}\left(G \wedge_{Y}\right.$ $\left.H^{c l_{Y}}\right)$. Then, $\varphi(z) \in G \wedge_{Y} H^{c l_{Y}}$, and thus there exist $g \in G$ and $h \in H^{c l_{Y}}$ such that $\varphi(z)=g \wedge h$. Since $\varphi$ is an isomorphism, we have

$$
z=\varphi^{-1}(\varphi(z))=\varphi^{-1}\left(g \wedge_{Y} h\right)=\varphi^{-1}(g) \wedge_{X} \varphi^{-1}(h) \in \varphi^{-1}(G) \wedge_{X} \varphi^{-1}\left(H^{c l_{Y}}\right) .
$$

Hence, $\varphi^{-1}\left(G \wedge_{Y} H^{c_{Y}}\right)=\varphi^{-1}(G) \wedge_{X} \varphi^{-1}\left(H^{c l_{Y}}\right)$.

Lemma 10. If $\varphi: X \rightarrow Y$ is a isomorphism of lower $B C K$-semilattices, then $(\forall A, B \in \mathcal{I}(X))\left(\varphi\left(A \wedge_{X} B\right)=\right.$ $\left.\varphi(A) \wedge_{Y} \varphi(B)\right)$.

Proof. Since $A$ and $B$ are ideals, we have $A \wedge_{X} B=\left\{a \wedge_{X} b \mid a \in A\right.$ and $\left.b \in B\right\}$. In addition, since $\varphi$ is epimorphism, for every $a \in A$ and $b \in B$ by using Lemma 6 , we have $\varphi\left(a \wedge_{X} b\right)=\varphi(a) \wedge_{Y} \varphi(b)$. Thus,

$$
\begin{aligned}
\varphi\left(A \wedge_{X} B\right) & =\varphi\left(\left\{a \wedge_{X} b \mid a \in A \text { and } b \in B\right\}\right) \\
& =\left\{\varphi(a) \wedge_{Y} \varphi(b) \mid a \in A \text { and } b \in B\right\} \\
& =\varphi(A) \wedge_{Y} \varphi(B) .
\end{aligned}
$$

Theorem 3. Let $\varphi: X \rightarrow Y$ be an epimorphism of lower BCK-semilattices. Given a mapping $c l_{Y}: \mathcal{I}(Y) \rightarrow$ $\mathcal{I}(Y)$ and any ideal $A$ of $X$ with $\operatorname{ker}(\varphi) \subseteq A$, define a map $c l \overleftarrow{Y}$ on $\mathcal{I}(X)$ as follows:

$$
c l_{Y}^{\overleftarrow{Y}}: \mathcal{I}(X) \rightarrow \mathcal{I}(X), A \mapsto \varphi^{-1}\left(\varphi(A)^{c l_{Y}}\right)
$$

(1) If " $c l_{Y}$ " is a weak closure operation on $\mathcal{I}(Y)$, then " $c l_{Y}^{\leftarrow}$ " is a weak closure operation on $\mathcal{I}(X)$.

(2) If " $c l_{Y}$ " is a semi-prime map on $\mathcal{I}(Y)$, then " $c l_{Y}^{\leftarrow}$ " is a semi-prime map on $\mathcal{I}(X)$.

(3) If "cl $l_{Y}$ " is a meet map on $\mathcal{I}(Y)$ and $\varphi$ is an isomorphism, then "cl ${ }_{Y}^{\leftarrow}$ " is a meet map on $\mathcal{I}(X)$.

Proof. (1) Suppose that " $\mathrm{cl}_{Y}$ " is a weak closure operation on $\mathcal{I}(Y)$. Let $A$ be an ideal of $X$. Then, $\varphi(A)$ is an ideal of $Y$ by Lemma 7 , and thus $\varphi(A) \subseteq \varphi(A)^{c l_{Y}}$. It follows from Lemma 8 that

$$
A=\varphi^{-1}(\varphi(A)) \subseteq \varphi^{-1}\left(\varphi(A)^{c l_{Y}}\right)=A^{c l_{Y}^{\overleftarrow{Y}}} .
$$

Now, let $A$ and $B$ be ideals of $X$ such that $A \subseteq B$. Then, $\varphi(A) \subseteq \varphi(B)$, and thus $\varphi(A)^{c l_{Y}} \subseteq \varphi(B)^{c l_{Y}}$. It follows that

$$
A^{c l_{Y}^{\leftarrow}}=\varphi^{-1}\left(\varphi(A)^{c l_{Y}}\right) \subseteq \varphi^{-1}\left(\varphi(B)^{c l_{Y}}\right)=B^{c l_{Y}^{\leftarrow}} .
$$

Therefore, "cl$l_{Y}^{\overleftarrow{Y}}$ " is a weak closure operation on $\mathcal{I}(X)$.

(2) Suppose that " $c l_{Y}$ " is a semi-prime map on $\mathcal{I}(Y)$. For any ideals $A$ and $B$ of $X$, we have

$$
\begin{aligned}
\varphi(A) \wedge_{Y} \varphi(B)^{c l_{Y}} & \subseteq\left(\varphi(A) \wedge_{Y} \varphi(B)\right)^{c l_{Y}} \\
& =\left(\left\{\varphi(a) \wedge_{Y} \varphi(b) \mid a \in A, b \in B\right\}\right)^{c l_{Y}} \\
& =\left(\left\{\varphi\left(a \wedge_{X} b\right) \mid a \in A, b \in B\right\}\right)^{c l_{Y}} \\
& =\varphi\left(A \wedge_{X} B\right)^{c l_{Y}}
\end{aligned}
$$


by Lemma 6, which implies from Lemmas 8 and 9 that

$$
\begin{aligned}
A \wedge_{X} B^{c l_{Y}^{\leftarrow}} & =A \wedge_{X} \varphi^{-1}\left(\varphi(B)^{c l_{Y}}\right) \\
& =\varphi^{-1}(\varphi(A)) \wedge_{X} \varphi^{-1}\left(\varphi(B)^{c l_{Y}}\right) \\
& =\varphi^{-1}\left(\varphi(A) \wedge_{Y} \varphi(B)^{c l_{Y}}\right) \\
& \subseteq \varphi^{-1}\left(\varphi\left(A \wedge_{X} B\right)^{c l_{Y}}\right) \\
& =\left(A \wedge_{X} B\right)^{c l_{Y}^{\leftarrow}} .
\end{aligned}
$$

Therefore, " $c l_{Y}^{\leftarrow}$ " is a semi-prime map on $\mathcal{I}(X)$.

(3) Suppose that " $c l_{Y}$ " is a meet map on $\mathcal{I}(Y)$ and $\varphi$ is an isomorphism. For any ideal $A$ of $X$ and $z \in X \backslash Z(X)$, we know that $\varphi(z)$ is a non-zeromeet element of $Y$ by Theorem 2. Using Lemmas 6, 8, and 10, we have

$$
\begin{aligned}
\left(z \wedge_{X} A\right)^{c l_{Y}^{\leftarrow}} & =\varphi^{-1}\left(\varphi\left(z \wedge_{X} A\right)^{c l_{Y}}\right) \\
& =\varphi^{-1}\left(\left(\varphi(z) \wedge_{Y} \varphi(A)\right)^{c l_{Y}}\right) \\
& =\varphi^{-1}\left(\varphi(z) \wedge_{Y} \varphi(A)^{c l_{Y}}\right) \\
& =\varphi^{-1}(\varphi(z)) \wedge_{X} \varphi^{-1}\left(\varphi(A)^{c l_{Y}}\right) \\
& =z \wedge_{X} A^{c l \overleftarrow{Y}} .
\end{aligned}
$$

Therefore, " $c l_{Y}^{\leftarrow}$ " is a meet $\operatorname{con} \mathcal{I}(X)$.

Corollary 1. If $\varphi: X \rightarrow Y$ is an isomorphism of lower BCK-semilattices and "cl $l_{Y}$ " is a prime weak closure operation on $\mathcal{I}(Y)$, then "cl $\stackrel{\leftarrow}{\text { " }}$ " is a prime weak closure operation on $\mathcal{I}(X)$.

Proof. The proof is straightforward.

Theorem 4. Let $\varphi: X \rightarrow Y$ be an epimorphism of lower $B C K$-semilattices. Given a mapping $c l_{X}: \mathcal{I}(X) \rightarrow$ $\mathcal{I}(X)$ and any ideal $B$ of $Y$, define a map $c l_{X}$ on $\mathcal{I}(Y)$ as follows:

$$
c l_{X}: \mathcal{I}(Y) \rightarrow \mathcal{I}(Y), B \mapsto \varphi\left(\varphi^{-1}(B)^{c l_{X}}\right) .
$$

(1) If " $c l_{X}$ " is a weak closure operation on $\mathcal{I}(X)$, then " $c l_{X}$ " is a weak closure operation on $\mathcal{I}(Y)$.

(2) If " $c l_{X}$ " is a semi-prime map on $\mathcal{I}(X)$, then " $c l_{X}{ }^{\prime}$ " is a semi-prime map on $\mathcal{I}(Y)$.

(3) If " $c l_{X}$ " is a meet map on $\mathcal{I}(X)$ and $\varphi$ is an isomorphism, then " $c l_{X}{ }^{\prime}$ " is a meet map on $\mathcal{I}(Y)$.

Proof. (1) Suppose that " $c l_{X}$ " is a weak closure operation on $\mathcal{I}(X)$ and let $A$ and $B$ be ideals of $Y$. Then, $\varphi^{-1}(A)$ and $\varphi^{-1}(B)$ are ideals of $X$ by Lemma 7. Since " $c l_{X}$ " is a weak closure operation on $\mathcal{I}(X)$, we have $\varphi^{-1}(A) \subseteq \varphi^{-1}(A)^{c l_{X}}$, which implies that

$$
A \subseteq \varphi\left(\varphi^{-1}(A)\right) \subseteq \varphi\left(\varphi^{-1}(A)^{c l_{X}}\right)=A^{c l} \vec{x} .
$$

Now, let $A \subseteq B$. Then, $\varphi^{-1}(A) \subseteq \varphi^{-1}(B)$, and thus $\varphi^{-1}(A)^{c l_{X}} \subseteq \varphi^{-1}(B)^{c l_{X}}$. It follows that

$$
A^{c l} \vec{X}=\varphi\left(\varphi^{-1}(A)^{c l_{X}}\right) \subseteq \varphi\left(\varphi^{-1}(B)^{c l_{X}}\right)=B^{c l} \vec{X} .
$$

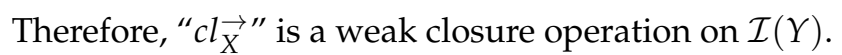

(2) Let " $c l_{X}$ " be a semi-prime map on $\mathcal{I}(X)$ and let $A$ and $B$ be ideals of $Y$. Then, we have

$$
\varphi^{-1}(A) \wedge_{X} \varphi^{-1}(B)^{c l_{X}} \subseteq\left(\varphi^{-1}(A) \wedge_{X} \varphi^{-1}(B)\right)^{c l_{X}} \subseteq \varphi^{-1}\left(A \wedge_{Y} B\right)^{c l_{X}}
$$


by Lemma 9. Hence,

$$
\varphi\left(\varphi^{-1}(A) \wedge_{X} \varphi^{-1}(B)^{c l_{X}}\right) \subseteq \varphi\left(\varphi^{-1}\left(A \wedge_{Y} B\right)^{c l_{X}}\right) .
$$

It follows from Lemma 10 that

$$
\begin{aligned}
A \wedge_{Y} B^{c l_{X}} & =A \wedge_{Y} \varphi\left(\varphi^{-1}(B)^{c l_{X}}\right) \\
& \subseteq \varphi\left(\varphi^{-1}(A)\right) \wedge_{Y} \varphi\left(\varphi^{-1}(B)^{c l_{X}}\right) \\
& =\varphi\left(\varphi^{-1}(A) \wedge_{X} \varphi^{-1}(B)^{c l_{X}}\right) \\
& \subseteq \varphi\left(\varphi^{-1}\left(A \wedge_{Y} B\right)^{c l_{X}}\right) \\
& =\left(A \wedge_{Y} B\right)^{c l_{X}} .
\end{aligned}
$$

Therefore, " $c l_{X}$ " is a semi-prime map on $\mathcal{I}(Y)$.

(3) Let " $c l_{X}$ " be a meet map on $\mathcal{I}(X)$ and assume that $\varphi$ is an isomorphism. Let $B \in \mathcal{I}(Y)$ and $z \in Y \backslash Z(Y)$. Then,

$$
\begin{aligned}
\left(z \wedge_{Y} B\right)^{c l_{X}} & =\varphi\left(\varphi^{-1}\left(z \wedge_{Y} B\right)^{c l_{X}}\right) \\
& =\varphi\left(\left(\varphi^{-1}(z) \wedge_{X} \varphi^{-1}(B)\right)^{c l_{X}}\right) \\
& =\varphi\left(\varphi^{-1}(z) \wedge_{X} \varphi^{-1}(B)^{c l_{X}}\right) \\
& =\varphi\left(\varphi^{-1}(z)\right) \wedge_{Y} \varphi\left(\varphi^{-1}(B)^{c l_{X}}\right) \\
& =z \wedge_{Y} B^{c l_{X}}
\end{aligned}
$$

Thus, " $c l \vec{X}$ " is a meet map on $\mathcal{I}(Y)$.

Corollary 2. If $\varphi: X \rightarrow Y$ is an isomorphism of lower BCK-semilattices and " $c l_{X}$ " is a prime weak closure operation on $\mathcal{I}(X)$, then " $\mathrm{cl}_{X}$ " is a prime weak closure operation on $\mathcal{I}(Y)$.

Proof. The proof is straightforward.

\section{Conclusions}

By using an epimorphism $\varphi: X \rightarrow Y$ of lower BCK-semilattices $X, Y$ and mapping $c l_{Y}: \mathcal{I}(Y) \rightarrow$ $\mathcal{I}(Y)$, and any ideal $A$ of $X$ with $\operatorname{ker}(\varphi) \subseteq A$, we define a new map $c l_{Y}^{\leftarrow}$ on $\mathcal{I}(X)$ by

$$
A \mapsto \varphi^{-1}\left(\varphi(A)^{c l_{Y}}\right) .
$$

and, moreover, we show that, if " $c l_{Y}$ " is a semi-prime, meet, and prime weak closure operation on $\mathcal{I}(Y)$, then so is "cl广⿳" on $\mathcal{I}(X)$.

Similarly, using a map $c l_{X}: \mathcal{I}(X) \rightarrow \mathcal{I}(X)$ and any ideal $B$ of $Y$, we define a new map $c l_{X}$ on $\mathcal{I}(Y)$ as follows:

$$
B \mapsto \varphi\left(\varphi^{-1}(B)^{c l_{X}}\right) .
$$

We prove that, if " $c l_{X}$ " is a semi-prime, meet, and prime weak closure operation on $\mathcal{I}(X)$, then so is

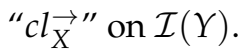

These results can be applied to investigate if the properties of closure operations are preserved under the homomorphism or not. In our future research, we will focus on other types of weak closure operations and we will study about homomorphic images and inverse images under homomorphism in different conditions. This kind of results can be useful to study isomorphism operations in different spaces. One of the motivations could be what happens for a decomposable ideal of a $B C K$-algebra under the homomorphism, or the associated prime ideals of a decomposable ideal under homomorphism. For more information about decompsable ideals and closure operations, please refer to [7]. 
In addition, other kinds of closure operations such as meet, tender, naive, etc. can be checked for homomorphic image and inverse image. For further information about other kinds of (weak) closure operation, please refer to [3,4]. Beside, for future research, we invite the researchers to join us and apply the results of this paper to concepts in [11-13] about the heigh of ideals. More precisely, we mean what happens for the height of ideals of $B C K$-algebra under homomorphisms.

Author Contributions: Conceived the idea, H.B. and Y.B.J.; literature review, H.B., and S.-Z.S.; writing一original draft preparation, H.B.; review and editing, H.B. and Y.B.J.; and Funding, S.-Z.S. All authors have read and agreed to the published version of the manuscript.

Funding: This research was supported by Basic Science Research Program through the National Research Foundation of Korea (NRF) funded by the Ministry of Education (No. 2016R1D1A1B02006812) and the 2020 scientific promotion program funded by Jeju National University.

Conflicts of Interest: The authors declare no conflict of interest.

\section{References}

1. Bordbar, H.; Zahedi, M.M.; Ahn, S.S.; Jun, Y.B. Weak closure operations on ideals of BCK-algebras. J. Comput. Anal. Appl. 2017, 23, 51-64.

2. Bordbar, H.; Jun, Y.B.; Zahedi, M.M. Tender and naive weak closure operations on lower BCK-semilattices. J. Comput. Anal. Appl. 2017, 25, 1354-1365.

3. Bordbar, H.; Zahedi, M.M. A finite type of closure operations on BCK-algebra. Appl. Math. Inf. Sci. Lett. 2016, 4, 1-9. [CrossRef]

4. Bordbar, H.; Zahedi, M.M. Semi-prime closure operations on BCK-algebra. Commun. Korean Math. Soc. 2015, 30, 385-402. [CrossRef]

5. Bordbar, H.; Zahedi, M.M.; Jun, Y.B. Semi-prime and meet weak closure operations in lower BCK-semilattices. Quasigruops Relat. Syst. 2017, 25, 41-50.

6. Bordbar, H.; Ahn, S.S.; Zahedi, M.M.; Jun, Y.B. Semiring structures based on meet and plus ideals in lower BCK-semilattices, J. Comput. Anal. Appl. 2017, 23, 945-954.

7. Bordbar, H.; Muhiuddin, G.; Alanazi, A.M. Primeness of Relative Annihilators in BCK-Algebra. Symmetry 2020, 12, 286. [CrossRef]

8. Bordbar, H.; Zahedi, M.M.; Jun, Y.B. Relative annihilators in lower BCK-semilattices. Math.Sci. Lett. 2017, 6, 1-7. [CrossRef]

9. Huang, Y. BCI-algebra; Science Press: Beijing, China, 2006.

10. Meng, J.; Jun, Y.B. BCK-algebras; Kyung Moon Sa Co.: Seoul, Korea, 1994.

11. Bordbar, H.; Cristea, I. Height of prime hyperideals in Krasner hyperrings. Filomat 2017, 31, $6153-6163$. [CrossRef]

12. Bordbar, H.; Cristea, I.; Novak, M. Height of hyperideals in Noetherian Krasner hyperrings. Univ. Politeh. Buchar. Sci. Bull. Ser. A Appl. Math. Phys. 2017, 79, 31-42.

13. Bordbar, H.; Novak, M.; Cristea, I. A note on the support of a hypermodule. J. Algebra and Appl. 2020, 19. [CrossRef]

(C) 2020 by the authors. Licensee MDPI, Basel, Switzerland. This article is an open access article distributed under the terms and conditions of the Creative Commons Attribution (CC BY) license (http://creativecommons.org/licenses/by/4.0/). 